INPLASY

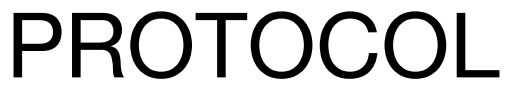

To cite: Lu et al. Chinese medicine as an adjunctive treatment for gastric cancer: methodological investigation of meta-analyses and evidence map. Inplasy protocol

202190005. doi:

10.37766/inplasy2021.9.0005

Received: 01 September 2021

Published: 01 September 2021

Corresponding author:

Cuncun Lu

cuncunlu2017@163.com

Author Affiliation:

Evidence-Based Medicine

Center, School of Basic

Medical Sciences, Lanzhou

University.

Support: None.

Review Stage at time of this submission: Preliminary

searches.

Conflicts of interest:

None declared.

\section{Chinese medicine as an adjunctive treatment for gastric cancer: methodological investigation of meta-analyses and evidence map}

Lu, C1; Ke, L2; Wang, Y3; Yang, K4.

Review question / Objective: 1. How about the methodological quality of meta-analyses regarding traditional Chinese medicine as an adjunctive treatment for gastric cancer? 2. What is the evidence of efficacy and safety of traditional Chinese medicine as an adjunctive treatment for gastric cancer in published meta-analyses?

Condition being studied: Clinical decision-making requires strong evidence based on weighing benefits and harms. traditional Chinese medicine has been used widely and many meta-analyses of traditional Chinese medicine for gastric cancer published recently, but efficacy and safety of traditional Chinese medicine and the methodological quality of published meta-analyses remained unknown. Therefore, we designed an overview to address this gap.

INPLASY registration number: This protocol was registered with the International Platform of Registered Systematic Review and Meta-Analysis Protocols (INPLASY) on 01 September 2021 and was last updated on 01 September 2021 (registration number INPLASY202190005).

\section{INTRODUCTION}

Review question / Objective: 1. How about the methodological quality of metaanalyses regarding traditional Chinese medicine as an adjunctive treatment for gastric cancer? 2. What is the evidence of efficacy and safety of traditional Chinese medicine as an adjunctive treatment for gastric cancer in published meta-analyses?

Condition being studied: Clinical decisionmaking requires strong evidence based on weighing benefits and harms. traditional Chinese medicine has been used widely and many meta-analyses of traditional Chinese medicine for gastric cancer 
published recently, but efficacy and safety of traditional Chinese medicine and the methodological quality of published metaanalyses remained unknown. Therefore, we designed an overview to address this gap.

\section{METHODS}

Search strategy: \#1 /OR "Medicine, Chinese Traditional"[Mesh] "Complementary Therapies"[Mesh] "Chinese materia medica"[Title/Abstract] "Chinese medicine"[Title/Abstract] "Chinese medicines"[Title/Abstract] "Traditional medicine"[Title/Abstract] "Traditional medicines"[Title/Abstract] herb*[Title/Abstract] pill*[Title] formula*[Title] granule *[Title] injection*[Title] decoction[Title] Wan[Title] San[Title] Gao[Title] Pian[Title] Dan[Title] Yin[Title] Ji[Title] \#2 /OR "Stomach Neoplasms"[Mesh] "gastric cancer"[Title/ Abstract] "gastric cancers"[Title/Abstract] "gastric carcinoma"[Title/Abstract] "gastric carcinomas"[Title/Abstract] "gastric adenocarcinoma"[Title/Abstract] "gastric adenocarcinomas"[Title/Abstract] "gastric neoplasm"[Title/Abstract] "gastric neoplasms"[Title/Abstract] "gastric tumour"[Title/Abstract] "gastric tumours"[Title/Abstract] "stomach cancer"[Title/Abstract] "stomach cancers"[Title/Abstract] "stomach carcinoma"[Title/Abstract] "stomach carcinomas"[Title/Abstract] "stomach adenocarcinoma"[Title/Abstract] "stomach adenocarcinomas" [Title/Abstract] "stomach neoplasm"[Title/Abstract] "stomach neoplasms"[Title/Abstract] "stomach tumor"[Title/Abstract] "stomach tumors"[Title/Abstract] \#3 /OR "Systematic Review"[Publication Type] "Systematic Reviews as topic"[Mesh] "Metaanalysis"[Publication Type] "Meta-analysis as topic"[Mesh] "systematic review"[Title/ Abstract] "meta-analysis"[Title/Abstract] \#4 \#1 AND \#2 AND \#3.

Participant or population: Gastric cancer treated by traditional Chinese medicine.

Intervention: Traditional Chinese medicine.
Comparator: Any other treatments (e.g., drugs) mentioned in included metaanalyses.

Study designs to be included: We will include published English meta-analyses focusing on traditional Chinese medicine as an adjunctive treatment for gastric cancer.

Eligibility criteria: We will include published English meta-analyses focusing on traditional Chinese medicine as an adjunctive treatment for gastric cancer.

Information sources: The meta-analyses that evaluated the efficacy and safety of traditional Chinese medicine as an adjunctive treatment for gastric cancer were collected via PubMed and Embase on 1st Sep, 2021 using a comprehensive search strategy developed using Medical Subject Headings and keywords, such as "Chinese medicine", "herb*", "decoction", "gastric cancer", "stomach neoplasm", "systematic review", "meta-analysis", etc.

Main outcome(s): 1.The efficacy and safety outcomes of traditional Chinese medicine for gastric cancer. 2.The methodological quality of meta-analyses regarding traditional Chinese medicine for gastric cancer.

Quality assessment / Risk of bias analysis: Two independent reviewers will employ the AMSTAR-2 to assess each included metaanalysis, and any disagreements will be resolved by consensus. Three options including "Yes," "Partial Yes," or "No" will be used to answer questions.

Strategy of data synthesis: The efficacy and safety data concerning traditional Chinese medicine as an adjunctive treatment for gastric cancer reported in meta-analyses will be summarized descriptively. The compliance rate of AMSTAR-2 will be calculated, including the numbers and their percentage of "Yes", "Partial Yes", or "No", with $95 \% \mathrm{Cls}$. The evidence mapping method will be used to present the results of quality assessment. Stata 17/SE will be used to analyze data. 
Subgroup analysis: None.

Sensitivity analysis: None.

Language: English.

Country(ies) involved: China.

Keywords: Chinese medicines; Gastric cancer; Meta-analyses; Methodological Quality; AMSTAR-2.

Contributions of each author:

Author 1 - Cuncun Lu.

Email: cuncunlu2017@163.com

Author 2 - Lixin Ke.

Email: kelx@mail2.sysu.edu.cn

Author 3 - Youyou Wang.

Email: youyouwang989@gmail.com

Author 4 - Kehu Yang.

Email: yangkh-ebm@Izu.edu.cn 